\title{
Standing sausage modes in curved coronal slabs
}

\author{
D. J. Pascoe and V. M. Nakariakov
}

\begin{abstract}
Centre for Fusion, Space and Astrophysics, Department of Physics, University of Warwick, CV4 7AL, UK
e-mail: D. J .Pascoe@warwick.ac.uk
\end{abstract}

Received 18 May 2015 / Accepted 6 July 2016

\begin{abstract}
Context. Magnetohydrodynamic waveguides such as dense coronal loops can support standing modes. The ratios of the periods of oscillations for different longitudinal harmonics depend on the dispersive nature of the waveguide and so may be used as a seismological tool to determine coronal parameters.

Aims. We extend models of standing sausage modes in low $\beta$ coronal loops to include the effects of loop curvature. The behaviour of standing sausage modes in this geometry is used to explain the properties of observed oscillations that cannot be accounted for using straight loop models.

Methods. We perform 2D numerical simulations of an oscillating coronal loop, modelled as a dense slab embedded in a potential magnetic field. The loop is field-aligned and so experiences expansion with height in addition to being curved. Standing sausage modes are excited by compressive perturbations of the loop and their properties are studied.

Results. The spatial profiles of standing sausage modes are found to be modified by the expanding loop geometry typical for flaring loops and modelled by a potential magnetic field in our simulations. Longitudinal harmonics of order $n>1$ have anti-nodes that are shifted towards the loop apex and the amplitude of anti-nodes near the loop apex is smaller than those near the loop footpoints.

Conclusions. We find that the observation of standing sausage modes by the Nobeyama Radioheliograph in a flaring coronal loop on 12 January 2000 is consistent with interpretation in terms of the global mode $(n=1)$ and third harmonic $(n=3)$. This interpretation accounts for the period ratio and spatial structure of the observed oscillations.
\end{abstract}

Key words. magnetohydrodynamics (MHD) - Sun: atmosphere - Sun: corona - Sun: magnetic fields - Sun: oscillations - waves

\section{Introduction}

Fast magnetohydrodynamic (MHD) waves are highly dispersive in waveguides such as coronal loops. For propagating modes generated by an impulsive driver, dispersion leads to the generation of quasi-periodic wave trains. This process was described by Roberts et al. (1983, 1984), Murawski \& Roberts (1994), Nakariakov \& Roberts (1995) and later observed with instruments such as the Solar Eclipse Coronal Imaging System (SECIS; Katsiyannis et al. 2003; Cooper et al. 2003) and the Atmospheric Imaging Assembly (AIA) on board the Solar Dynamics Observatory (SDO; e.g. Shen \& Liu 2012; Liu et al. 2012; Yuan et al. 2013). The dispersion arises owing to the presence of a characteristic transverse length scale and occurs for both sausage $(m=0)$ and kink $(m=1)$ fast modes. For example, Oliver et al. (2014) recently studied the dispersive evolution of propagating kink waves in coronal loops. The dispersion of propagating fast MHD waves has also been considered for current sheets (Edwin et al. 1986; Jelínek \& Karlický 2012; Jelínek et al. 2012), coronal holes (Pascoe et al. 2014), and flaring active regions (Nisticò et al. 2014; see also recent review by Pascoe 2014).

For standing modes, the effect of dispersion is apparent in the ratio of periods for different longitudinal harmonics. For a standing mode of wavelength $\lambda$ in a loop of length $L$ the footpoints are nodes for the oscillation and so the longitudinal wavenumber $k$ must be an integer multiple of $\pi / L$, i.e.

$k_{n}=\frac{n \pi}{L}$, where $n$ is the order of the harmonic. The global or fundamental standing mode is $n=1$, while the $n=2$ mode is the first overtone, or second harmonic, and so on for higher order modes. The period of oscillation for each mode may be denoted as $P_{n}$. Andries et al. (2009) point out in their review of coronal seismology using kink overtones that the term "harmonic" should strictly be used in the dispersionless case of overtone frequencies being exact multiples of the fundamental frequency. For clarity we refer to the different standing modes using their order $n$, which corresponds to the number of anti-nodes in the longitudinal spatial profile of the mode. The " $n$th harmonic" therefore more generally refers to the oscillation with $n$ anti-nodes.

Owing to dispersion, the period ratio $P_{1} / 2 P_{2}$ is less than unity for fast MHD waves. Andries et al. (2005) considered the case of kink waves in long loops, for which the thin tube approximation removes the effect of dispersion. The period ratio was calculated to again be $P_{1} / 2 P_{2}<1$ when longitudinal density structuring was introduced, enabling simultaneous observations of the multiple harmonics (e.g. Verwichte et al. 2004) to be used as a seismological tool for determining the density scale height. McEwan et al. (2006) demonstrated that longitudinal structuring has the dominant effect on the period ratio and calculated the density scale height of two of the loops reported in Verwichte et al. (2004). McEwan et al. (2008) derived an analytical expression for the dependence of the period ratio on the density scale height, which was previously solved numerically by Donnelly et al. (2006) and Díaz et al. (2007) for loops, and by Donnelly et al. (2007) for a magnetic arcade. A spatially resolved observation of the fundamental and second harmonic standing kink modes in a coronal loop was recently reported by 
Pascoe et al. (2016), with a period ratio $P_{1} / 2 P_{2}=1.15 \pm 0.22$. The ratio being greater than unity suggests a negative scale height, which was also considered by Andries et al. (2005) for coronal loops and Lomineishvili et al. (2014) for prominence threads.

For straight models of coronal loops (without longitudinal variations of parameters along $z$ ), standing modes are described using sinusoidal profiles of the form $\sin \left(k_{n} z\right)$. For these types of profiles the nodes and anti-nodes are equidistant. This is no longer true when additional effects such as longitudinal stratification or divergence are included. In these cases, the longitudinal wavenumber $k_{n}$ represents an average value and the positions of the nodes and anti-nodes are shifted in space in comparison with sinusoids. Observations of these shifts could potentially be used for spatial magneto-seismology since they depend on, for example, the density stratification (Erdélyi \& Verth 2007; Verth et al. 2007), and longitudinal inhomogeneities (Verth \& Erdélyi 2008) for transversal coronal loop oscillations. These types of shifts have also been investigated for torsional Alfvén waves (Verth et al. 2010) and spicules (Verth et al. 2011).

In contrast with the standing kink modes commonly observed in long coronal loops (e.g. Aschwanden et al. 1999; Nakariakov et al. 1999), standing sausage modes are expected to be found only in short, dense loops, of which there are fewer examples (Aschwanden et al. 2004). This is due to the existence of a cut-off wavelength (e.g. Roberts et al. 1983) which limits the minimum aspect ratio $a / L$ and density contrast $\rho_{0} / \rho_{\mathrm{e}}$ for the fundamental standing sausage mode to be supported as a trapped mode (Nakariakov et al. 2003). When these constraints are not satisfied, the sausage mode is leaky and can quickly decay (e.g. Cally 1986; Pascoe et al. 2007b), with a period and damping rate that has recently been analytically derived by Vasheghani Farahani et al. (2014).

Pascoe et al. (2009b) performed numerical simulations of sausage oscillations in loops with a non-uniform cross-section as a model for flaring loops that expand near the apex owing to heating. The period of the global sausage mode $\left(P_{1}\right)$ and the third harmonic $\left(P_{3}\right)$ were considered and were found to be most strongly determined by the minimum loop width. Strong divergence was also found to lead to the coupling of the longitudinal harmonics. The period ratio has also been shown to depend on loop expansion for the case of standing slow modes (Luna-Cardozo et al. 2012).

The effect of flows on the behaviour of standing sausage modes has been investigated by Li et al. $(2013,2014)$ who found that they increase the minimum aspect ratio required for trapped standing sausage modes to exist. However, the flows typically only reduced the period ratio $P_{1} / 2 P_{2}$ by less than about $5 \%$.

The effect of curvature on coronal loop oscillations has been previously investigated by a number of authors (see review by van Doorsselaere et al. 2009). Studies have demonstrated that curved loops require a non-uniform equilibrium magnetic field and consequently may introduce an additional damping mechanism for oscillations in the form of lateral wave leakage by tunnelling of wave energy (e.g. Smith et al. 1997; Brady et al. 2006; Verwichte et al. 2006b). We note however that in our model (Sect. 3) this mechanism is prohibited by our choice of Alfvén speed profile (and the loop is sufficiently dense and thick to be in the trapped regime).

Goossens et al. (2009) discuss how damping owing to resonant absorption dominates over that by leakage for kink waves in straight loops with a finite inhomogeneous layer (see also Goossens \& Hollweg 1993). Terradas et al. (2006) performed simulations of curved coronal loops and found that the effect of resonant absorption remains stronger than lateral leakage. Curvature is found to be unimportant for standing kink modes because of their small aspect ratio $L \gg a$. However curvature might be more important for sausage modes due to the condition that they require sufficiently thick and dense loops to exist as trapped modes.

Nakariakov et al. (2003) interpret the observation of quasiperiodic pulsations by the Nobeyama Radioheliograph (NoRH) in terms of standing sausage modes. The oscillations were measured in a flaring coronal loop on 12 January 2000. The spatial resolution of the observations allows two periodicities with different spatial profiles to be identified. It was proposed that these correspond to the global standing mode and the second harmonic. However, this interpretation implies a large period ratio $P_{1} / 2 P_{2} \approx 0.8$ which has not been accounted for. Melnikov et al. (2005) considered the same observation and proposed a number of alternative candidates for the shorter period mode, including the third longitudinal harmonic of the sausage mode. Observations of multiple sausage oscillations in a cool post-flare loop have also been reported by Srivastava et al. (2008).

In this paper we consider the effect of curvature and loop expansion on the behaviour of standing sausage modes using 2D numerical simulations of dense slabs embedded in a potential magnetic field. In particular, we consider the effect on the period ratios and spatial profiles of the modes because of their potential seismological application. In Sect. 2 we first review effects of coronal loop parameters on the period ratio in the case of straight coronal loops. We note, however, that these effects alone are insufficient to account for the observations by Nakariakov et al. (2003). In Sect. 3 we then present our results of numerical simulations of standing sausage modes in curved and expanding coronal slabs. Discussion of our results with regards to the observations of Nakariakov et al. (2003) and concluding remarks are presented in Sect. 4.

\section{Parametric study of period ratios}

Here we discuss several parameters which are known to have an effect on the period ratio of standing modes in coronal loops modelled as straight waveguides.

\subsection{Loop geometry and aspect ratio}

Figure 1 shows the phase speed $\omega / k$ of trapped modes as a function of normalised wavenumber $k a$. The solid lines corresponds to the slab geometry (Edwin \& Roberts 1982) and the dashed lines to the cylindrical geometry (Edwin \& Roberts 1983). The sausage mode is weakly dependent on the choice of geometry in comparison to the kink mode in the case of loops modelled as straight slabs or cylinders. For both cylindrical and slab geometries the trapped sausage mode phase speed is limited by $C_{\mathrm{A} 0}$ at $k a \rightarrow \infty$ and increases to $C_{\mathrm{Ae}}$ at $k_{\mathrm{c}} a$ where $k_{\mathrm{c}}$ is the cut-off wavenumber. In the long wavelength limit the kink mode also has a phase speed $C_{\mathrm{Ae}}$ for the slab geometry, but for the cylindrical geometry tends to the kink speed $C_{k}$ (middle dotted line).

The existence of the sausage mode cut-off in the long wavelength limit means observations with a large Q-factor (i.e. a large number of oscillation cycles) require sufficiently thick and dense coronal loops so that standing modes fall into the trapped regime. The density contrast of $\rho_{0} / \rho_{\mathrm{e}}=50$ used in Fig. 1 (and subsequent figures) satisfies this condition, and is taken from Nakariakov et al. (2003) who use this estimate for their 
D. J. Pascoe and V. M. Nakariakov: Sausage modes in curved slabs
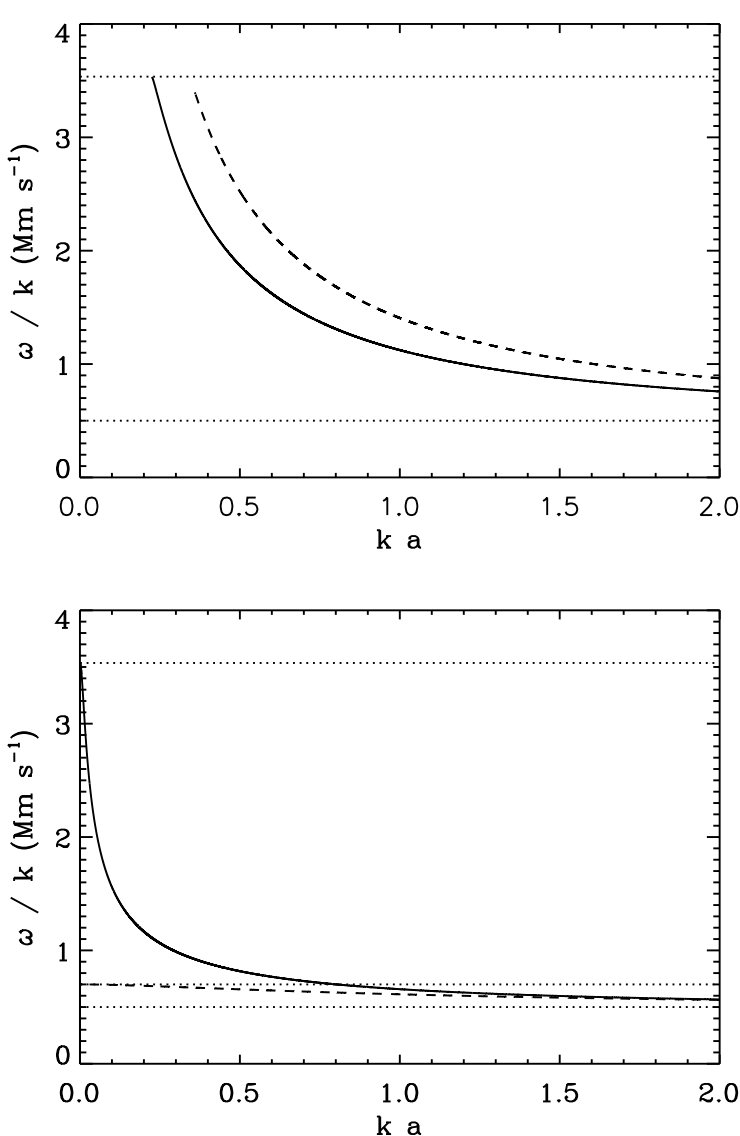

Fig. 1. Phase speed $\omega / k$ as a function of normalised wavenumber $k a$ for the trapped sausage (top) and kink (bottom) modes in a loop with density contrast $\rho_{0} / \rho_{\mathrm{e}}=50$ (and plasma $\beta=0$ ). The solid lines are for the slab geometry and the dashed lines are for the cylindrical geometry. The lower and upper dotted lines correspond to the internal Alfvén speed $C_{\mathrm{A} 0}$ and external Alfvén speed $C_{\mathrm{Ae}}$, respectively. In the lower panel the middle dotted line corresponds to the kink speed $C_{k}$.

interpretation of NoRH observations. The effect of varying the density contrast is considered in Sect. 2.2. The requirement of a sufficiently large loop aspect ratio $a / L$ for trapped sausage modes also introduces the effect of geometrical dispersion so that the period ratios of the standing modes depend on the loop parameters and may therefore be used for seismological purposes. Figure 2 shows the period ratios $P_{1} / 2 P_{2}$ and $P_{1} / 3 P_{3}$ as a function of the loop aspect ratio for sausage modes in slab and cylinder geometries. The effect of dispersion is stronger for wavenumbers closer to the cut-off.

Macnamara \& Roberts (2011) produced analytical expressions for the period ratio $P_{1} / 2 P_{2}$ of the sausage and kink modes in a magnetic slab. For the transverse density structure they considered an Epstein profile (Eq. (7) with $p=1$ ). They give the square of the wave speed for the sausage mode in their Eq. (32) as

$$
\begin{aligned}
c^{2}= & \frac{C_{\mathrm{A} 0}^{2}}{2 C_{\mathrm{Ae}}^{2} k^{2} a^{2}}\left(2 C_{\mathrm{Ae}}^{2} k^{2} a^{2}+4 C_{\mathrm{Ae}}^{2}-9 C_{\mathrm{A} 0}^{2}\right. \\
& \left.+3 \sqrt{9 C_{\mathrm{A} 0}^{4}-4 C_{\mathrm{A} 0}^{2} C_{\mathrm{Ae}}^{2} k^{2} a^{2}-8 C_{\mathrm{A} 0}^{2} C_{\mathrm{Ae}}^{2}+4 C_{\mathrm{Ae}}^{4} k^{2} a^{2}}\right) .
\end{aligned}
$$

This can be used to calculate the period ratio. Macnamara \& Roberts (2011) define the loop length to be

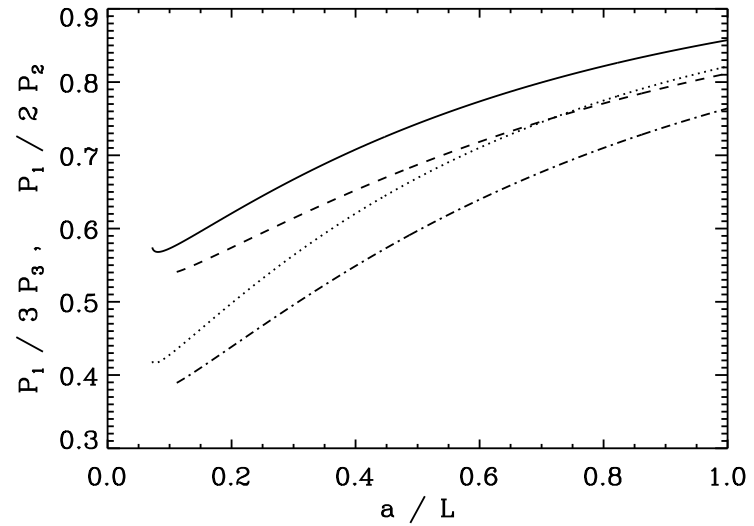

Fig. 2. Period ratio $P_{1} / 2 P_{2}$ for slab (solid) and cylinder (dashed) geometries and $P_{1} / 3 P_{3}$ for slab (dotted) and cylinder (dot-dashed).

$2 L$ whereas we use $L$, so our equivalent of their Eq. (33) is

$$
\begin{aligned}
\left(\frac{P_{1}}{2 P_{2}}\right)^{2} & =\left(\frac{c_{2}}{c_{1}}\right)^{2} \\
& =\frac{1}{4}\left(\frac{4+8 \epsilon-9 \chi+3 \sqrt{9 \chi^{2}-16 \chi \epsilon-8 \chi+16 \epsilon}}{4+2 \epsilon-9 \chi+3 \sqrt{9 \chi^{2}-4 \chi \epsilon-8 \chi+4 \epsilon}}\right),
\end{aligned}
$$

where $\epsilon=(\pi a / L)^{2}$ and $\chi=\left(C_{\mathrm{A} 0} / C_{\mathrm{Ae}}\right)^{2}$. If we consider the limit of high density contrast we have $\chi \rightarrow 0$ and the approximation

$\frac{P_{1}}{2 P_{2}}=\left(\frac{1+2 \epsilon+3 \sqrt{\epsilon}}{4+2 \epsilon+6 \sqrt{\epsilon}}\right)^{1 / 2}$.

Since this approximation excludes the effects of a finite density contrast it no longer describes the sausage mode cut-off. In the limit of the aspect ratio $a / L \rightarrow 0$ the effect of geometrical dispersion is also removed and the period ratio $P_{1} / 2 P_{2} \rightarrow 1 / 2$ as in Macnamara \& Roberts (2011). Similarly, for the ratio $P_{1} / 3 P_{3}$ we obtain

$\left(\frac{P_{1}}{3 P_{3}}\right)^{2}=\frac{1}{4}\left(\frac{4+18 \epsilon-9 \chi+3 \sqrt{9 \chi^{2}-36 \chi \epsilon-8 \chi+36 \epsilon}}{4+2 \epsilon-9 \chi+3 \sqrt{9 \chi^{2}-4 \chi \epsilon-8 \chi+4 \epsilon}}\right)$,

and for $\chi \rightarrow 0$ the approximation

$\frac{P_{1}}{3 P_{3}}=\left(\frac{4 / 9+2 \epsilon+2 \sqrt{\epsilon}}{4+2 \epsilon+6 \sqrt{\epsilon}}\right)^{1 / 2}$,

which tends to $1 / 3$ in the limit $a / L \rightarrow 0$.

Figure 3 shows period ratios as a function of aspect ratio for sausage modes in a slab geometry with an Epstein density profile. The behaviour is similar to that for the slab with the step function profile (Fig. 2). The symbols represent ratios calculated from the periods of the relevant harmonics, while the lines represent the derived expressions and approximations discussed above.

\subsection{Density profile steepness and contrast ratio}

In the previous section the density profile was taken to be either a step function, switching discontinuously from its internal value $\rho_{0}$ to its external value $\rho_{\mathrm{e}}$ at the loop radius $x=a$, or the Epstein profile considered by Macnamara \& Roberts (2011). It has recently been established that the transverse profile steepness affects the period of sausage modes quite significantly 


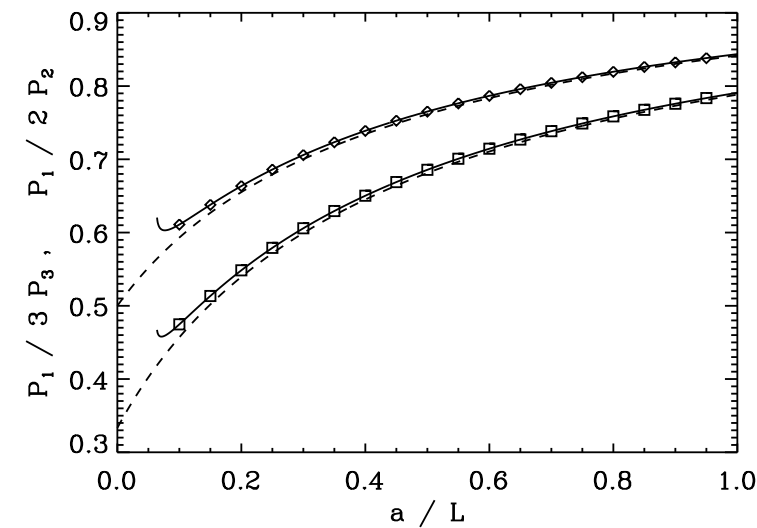

Fig. 3. Period ratios $P_{1} / 2 P_{2}$ (triangles) and $P_{1} / 3 P_{3}$ (squares) as a function of the aspect ratio $a / L$. The symbols represent numerical calculations. The solid lines correspond to the analytical expressions for the ratios given by Eqs. (3) and (5), while the dashed lines represent approximations for a high density contrast given by Eqs. (4) and (6).

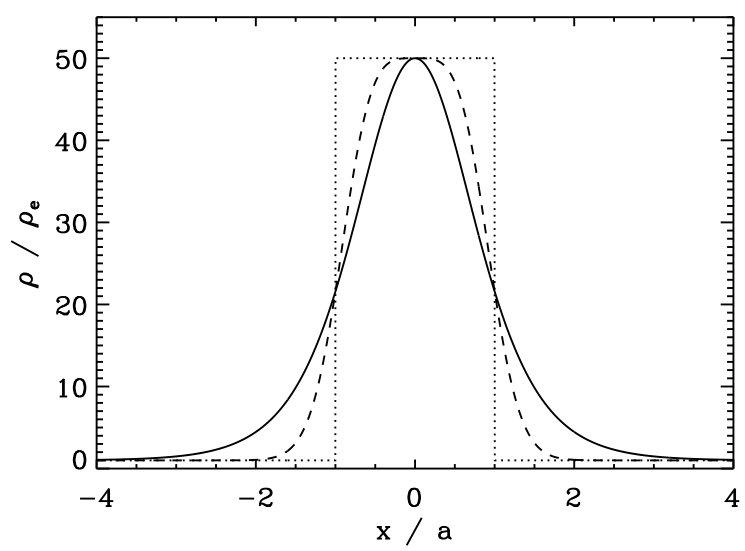

Fig. 4. Density profiles for a loop with contrast ratio $\rho_{0} / \rho_{\mathrm{e}}=50$ and different steepness parameters $p$. The solid line is the Epstein profile $(p=1)$. The dashed line corresponds to $p=2$, while the dotted line is a step function profile $(p \rightarrow \infty)$

(Nakariakov et al. 2012). Here we consider the effect of various smooth density profiles in more detail. A convenient density profile for this study is the generalised symmetric Epstein profile (e.g. Nakariakov \& Roberts 1995)

$\rho(x)=\left(\rho_{0}-\rho_{\mathrm{e}}\right) \operatorname{sech}^{2}\left[\frac{|x|}{a}\right]^{p}+\rho_{\mathrm{e}}$,

where $\rho_{0}$ is the density at the loop axis, $\rho_{\mathrm{e}}$ is the external density at $x \rightarrow \infty$, the width of the loop is $2 a$, and $p$ is the steepness parameter. As a result of this profile, the Alfvén speed increases from a local minimum of $C_{\mathrm{A} 0}$ at the centre to $C_{\mathrm{Ae}}$ at infinity, and so forms a waveguide for fast MHD waves. Our monolithic slab model ignores the effect of fine structuring of the loop which may be present. NoRH observes flaring, hot loops for which multi-threaded structuring is not detected. Pascoe et al. (2007a) demonstrate that the period of oscillation for standing sausage modes is not affected by very fine structuring of the slab density profile.

Figure 4 shows examples of density profiles given by Eq. (7) for a loop with contrast ratio $\rho_{0} / \rho_{\mathrm{e}}=50$. The solid line is the Epstein profile with steepness parameter $p=1$ (e.g. Fig. 3). The dashed line corresponds to $p=2$, while the dotted line is a step function profile obtained for $p \rightarrow \infty$. Analytical solutions for

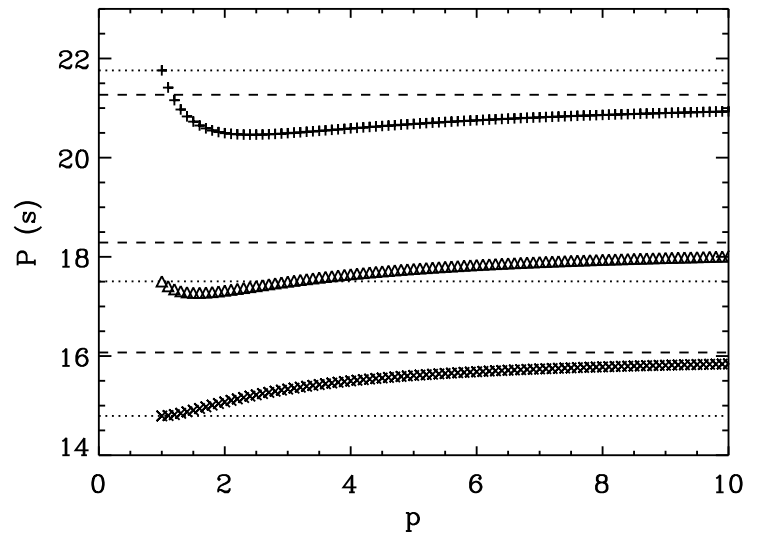

Fig. 5. Period of oscillation as a function of the density profile steepness parameter. $P_{1}, P_{2}$ and $P_{3}$ are represented by plus signs, triangles and crosses, respectively. The symbols represent numerical calculations. The dotted and dashed lines correspond to the analytical expressions for the Epstein and step profiles, respectively.

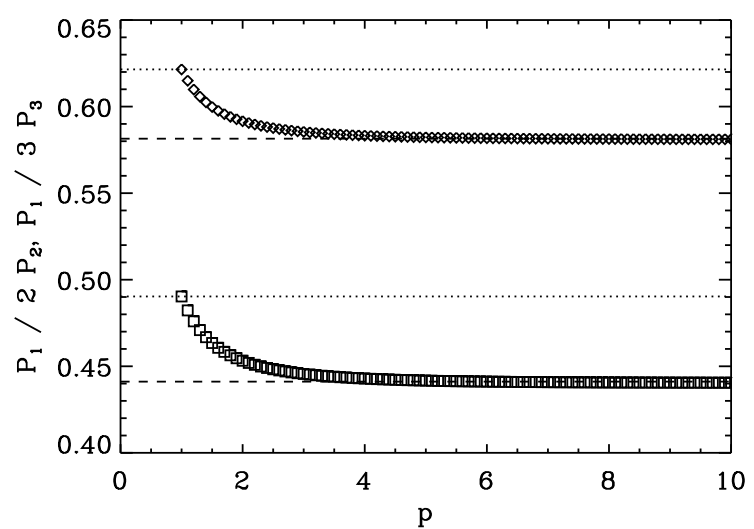

Fig. 6. Period ratios $P_{1} / 2 P_{2}$ (triangles) and $P_{1} / 3 P_{3}$ (squares) as a function of the density profile steepness parameter. The symbols represent numerical calculations. The dotted and dashed lines correspond to the analytical expressions for the Epstein and step profiles, respectively.

the dispersion relation are known for the step profile and for the Epstein profile with $\beta=0$. For all other $p$ the solutions must be calculated numerically. As in Pascoe et al. (2007a) we solve the sausage mode dispersion relation by the shooting method (based on a fourth-order Runge-Kutta technique).

Figure 5 shows the dependence of the period of oscillation for $n=1,2$, and 3 as a function of the density profile steepness parameter $p$. A loop length of $L=25 \mathrm{Mm}$ and density contrast $\rho_{0} / \rho_{\mathrm{e}}=50$ have been assumed. For increasing $p$, each period starts at the value for the Epstein profile and then tends, from below, to the value for a step profile. Figure 6 shows that the period ratios $P_{1} / 2 P_{2}$ and $P_{1} / 3 P_{3}$ tend to the value for the step profile more quickly than the individual periods do. The step function period ratios are therefore good approximations for profiles with steepness parameter $p \gtrsim 3$.

Next we consider the effect of varying the loop density contrast ratio $\rho_{0} / \rho_{\mathrm{e}}$. Figure 7 shows the period ratios $P_{1} / 2 P_{2}$ and $P_{1} / 3 P_{3}$ as a function of density contrast for $p=1$ (diamonds), $p=2$ (squares), and a step function density profile (crosses). The solid and dashed lines represent the analytical solutions for the Epstein and step function density profiles, respectively. In all cases the period ratios saturate to a constant value for $\rho_{0} / \rho_{\mathrm{e}} \gtrsim 50$. 


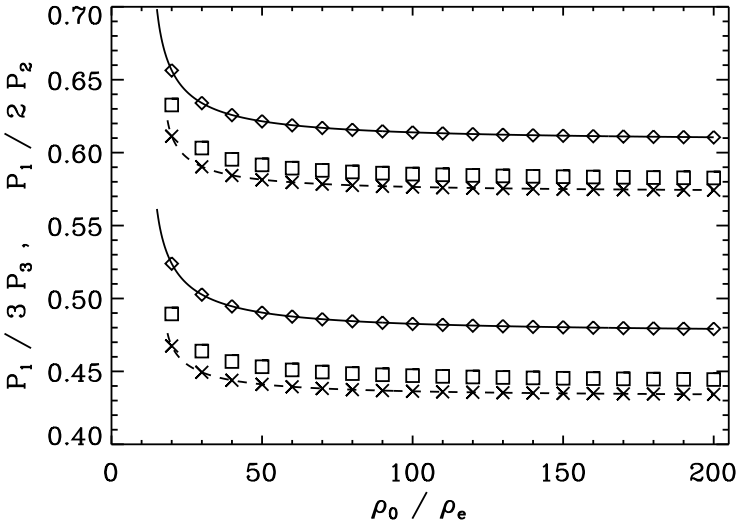

Fig. 7. Period ratios $P_{1} / 2 P_{2}$ and $P_{1} / 3 P_{3}$ as a function of the density contrast $\rho_{0} / \rho_{\mathrm{e}}$ for $p=1$ (diamonds), $p=2$ (squares) and a step function density profile (crosses). The solid and dashed lines represent the analytical solutions for the Epstein and step function density profiles, respectively.

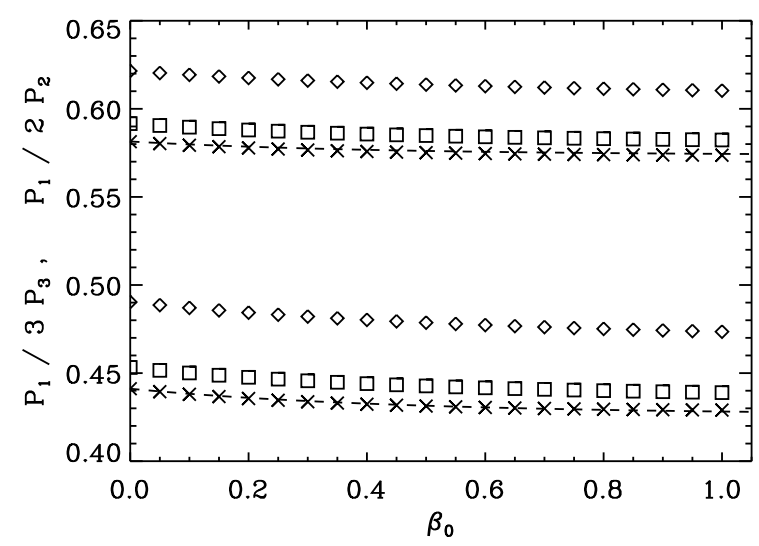

Fig. 8. Period ratios $P_{1} / 2 P_{2}$ and $P_{1} / 3 P_{3}$ as a function of the internal plasma beta $\beta_{0}$ for $p=1$ (diamonds), $p=2$ (squares), and a step function density profile (crosses). The dashed lines represent the analytical solutions for the step function density profile.

\subsection{Plasma beta}

A common approximation when studying fast MHD waves in the corona is a cold plasma, i.e. $\beta=0$. Here we consider the effect of a finite plasma beta which is particularly relevant for oscillations in hot flaring loops. The effect of finite plasma beta on the period of sausage modes was investigated by Inglis et al. (2009) who found that the period of the global sausage mode $\left(P_{1}\right)$ varied by less than $5 \%$ for $0<\beta<1$. An expression for the dependence of the cut-off wavenumber $k_{\mathrm{c}}$ on $\beta$ was derived for a magnetic slab with a step function profile, and this was also found to exhibit weak dependency for coronal loops having low values of external plasma beta.

Figure 8 shows the period ratios $P_{1} / 2 P_{2}$ and $P_{1} / 3 P_{3}$ as a function of the internal plasma beta $\beta_{0}$ for $p=1$ (diamonds), $p=2$ (squares) and a step function density profile (crosses). The dashed lines represent the analytical solutions for the step function density profile (the analytical solution for the Epstein profile is known for $\beta=0$ only). The period ratios are weakly dependent on the internal plasma beta which is consistent with the study by Inglis et al. (2009).

\section{Numerical simulations of standing sausage modes in a curved slab}

In this section we present results of $2 \mathrm{D}$ numerical simulations of standing sausage modes in curved and expanding coronal slabs. Simulations were performed using LARE2D (Arber et al. 2001) to solve the ideal MHD equations. The numerical domain is composed of $2000 \times 1000$ grid points. Results were checked by convergence tests using resolutions of $4000 \times 2000$ and $8000 \times 4000$, with no significant changes (e.g. periods of oscillation varying by less than $1 \%$ ). The boundary conditions are line-tied to allow reflections at the base of the corona. Reflections from other boundaries were removed using damping layers. In normalised units, the numerical domain has a size $2 \pi \times \pi$ and the runtime is $\tilde{t}=500$. The conversion of results to physical units is discussed in Sect. 3.1.

The magnetic field used in our model is a potential field (see also Nisticò et al. 2014) given by

$\boldsymbol{B}=B_{0} \exp \left(-y / l_{0}\right)\left[\cos \left(x / l_{0}\right) \overline{\boldsymbol{e}}_{x}-\sin \left(x / l_{0}\right) \overline{\boldsymbol{e}}_{y}\right]$,

where $B_{0}$ is a constant determining the magnitude of the field and $l_{0}$ is the normalisation factor for the spatial size. The density profile for the field-aligned coronal loop is based on the generalised symmetric Epstein profile (Eq. (7)) with additional modifications to take into account the loop curvature and expansion (the increase in width at the apex). The density profile is also stratified in the vertical direction with a scale height $\Lambda$;

$\rho=\left[\left(\rho_{0}-\rho_{\mathrm{e}}\right) \operatorname{sech}^{2}\left(\frac{r}{a(s)}\right)^{p}+\rho_{\mathrm{e}}\right] \exp \left(-\frac{y+\pi}{\Lambda}\right)$,

where $r$ is the distance from the loop axis (along the local equipotential line) and $a(s)$ is the local loop semi-width which is a function of the distance along the loop axis $s=[0, L]$.

The behaviour of MHD waves in a potential arcade model has also been considered by previous authors. For the case of an arcade with a constant Alfvén speed and a spatially periodic perturbation the analytic solution for fast waves was derived by Cadez et al. (1996). Smith et al. (1997) examined the effect of curvature on fast MHD waves and found that it caused leakage. Terradas et al. (1999) studied the influence of the vertical Alfvén speed profile and the nonpotentiality of the field on the behaviour of fast modes. In the case of the Alfvén speed decreasing with height, fast energy was again found to leak upwards which was also investigated by Smith et al. (1997), Verwichte et al. (2006a,b), and Brady et al. (2006). Verwichte et al. (2006c) used this model to develop a seismological tool to determine the transverse density structuring from observations of vertically polarised kink modes damped by lateral leakage. Oliver et al. (1998) performed numerical simulations of impulsively excited linear and non-linear fast MHD waves in a coronal arcade. Arregui et al. (2004) investigated the effect of mode coupling which is introduced when the arcade is sheared. The impulsive excitation of vertical kink oscillations of a dense loop in a potential arcade was modelled by Gruszecki \& Murawski (2008) while Gruszecki et al. (2008) simulated the influence of a photospheric layer with finite density rather than assuming line-tied boundary conditions. The damping rate of vertical kink oscillations was found to increase by this additional route for energy leakage.

The particular loop parameters we choose are based on the observations by Nakariakov et al. (2003) and so the density contrast ratio is taken to be $\rho_{0} / \rho_{\mathrm{e}}=50$ and aspect ratio $a / L=0.12$ at the loop apex (decreasing to $a / L=0.066$ at the loop footpoints). 

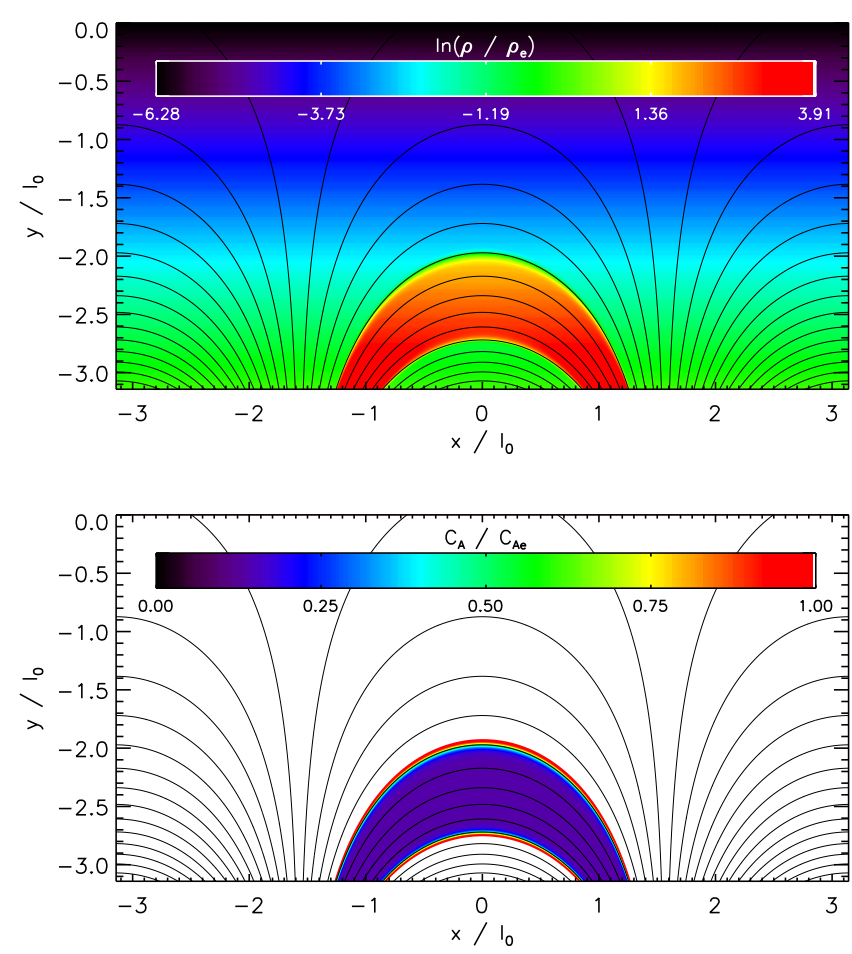

Fig. 9. Equilibrium used in our numerical simulations of standing sausage modes in curved coronal slabs. The line contours show the magnetic field lines. Top: the colour contours show the (logarithm of) density, which has an enhancement describing a curved field-aligned loop with density contrast ratio $\rho_{0} / \rho_{\mathrm{e}}=50$ and an aspect ratio of $a / L=0.12$ at its apex. Bottom: the colour contours show the Alfvén speed which is constant inside and outside the loop.

The density steepness parameter is taken to be $p=8$, which is a good approximation for the slab profile but remains numerically well-resolved. The plasma beta in the numerical simulations is taken to be small $\beta<10^{-3}$. However, the plasma beta itself is known to have a small effect of the period of oscillation (see Sect. 2.3). Our loop geometry may therefore be considered to represent models in which high beta plasma at the loop apex causes expansion of the magnetic field there (e.g. Zaitsev \& Stepanov 1982). The equilibrium magnetic field and density profiles are shown in Fig. 9. The line contours show the magnetic field lines while the colour contours show the density (top panel) and Alfvén speed (bottom panel). The coronal loop is defined as a field-aligned density enhancement with $\rho_{0} / \rho_{\mathrm{e}}=50$. The density varies with height in accordance with Eq. (9) and for simplicity we choose $\Lambda=0.5$ which ensures that the loop structure provides the only variation in Alfvén speed, i.e. the Alfvén speed is constant inside and outside the loop, only varying in the thin transition layer between the loop and the environment. This makes comparison of our loop model with an equivalent straight slab (Edwin \& Roberts 1982) easier and also removes the effect of leakage due to tunnelling present when the external Alfvén speed varies with height.

Standing sausage modes are excited by applying velocity perturbations which approximate the eigenmode, based on modifying the solutions for a straight coronal loop (e.g. Cooper et al. 2003). In the $r$-direction perpendicular to the loop axis, the transverse velocity $v_{r}$ is anti-symmetrical about the loop axis to excite (compressive) sausage waves. In the longitudinal direction $s$, the perturbation has a sinusoidal profile with a wavenumber corresponding to the harmonic we choose to generate. The radial velocity perturbation is decomposed into its Cartesian components

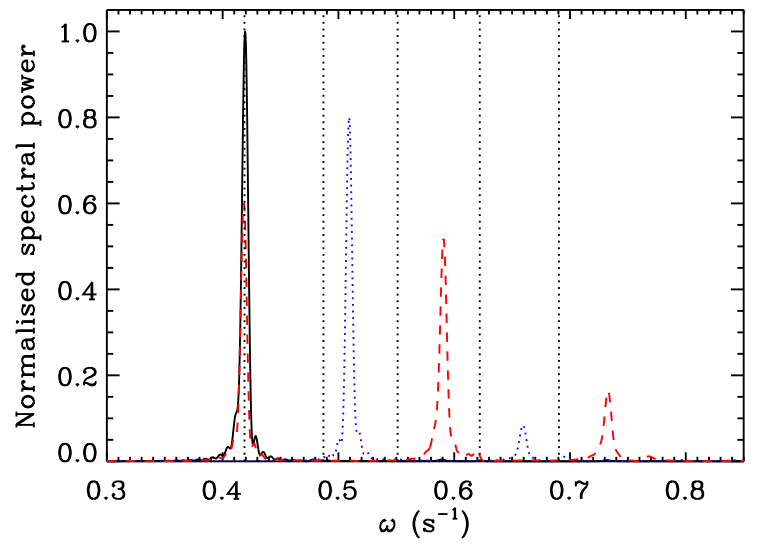

Fig. 10. Spectral power of sausage oscillations in a curved coronal loop, excited by drivers with $n=1$ (solid line), 2 (blue dotted line), and 3 (red dashed line). The vertical dotted lines correspond to the frequencies for $n=1-5$ calculated for a straight loop (Table 1 ).

Table 1. Period of oscillation (in seconds) of standing sausage modes from numerical simulations of curved coronal loops and the analytical calculation for a straight loop.

\begin{tabular}{ccc}
\hline \hline & Curved & Straight \\
\hline$P_{1}$ & 15.0 & 15.0 \\
$P_{2}$ & 12.3 & 12.9 \\
$P_{3}$ & 10.6 & 11.4 \\
$P_{4}$ & 9.5 & 10.1 \\
$P_{5}$ & 8.5 & 9.1 \\
\hline$P_{1} / 2 P_{2}$ & 0.61 & 0.58 \\
$P_{1} / 3 P_{3}$ & 0.47 & 0.44 \\
$P_{1} / 4 P_{4}$ & 0.40 & 0.37 \\
$P_{1} / 5 P_{5}$ & 0.35 & 0.33 \\
\hline
\end{tabular}

for use in LARE2D;

$v_{r}=A \frac{\sinh (r / a)}{\cosh ^{v}(r / a)} \operatorname{sgn}(x) \sin (n \pi s / L)$,

$v_{x}=v_{r} \sin \left(x / l_{0}\right)$,

$v_{y}=v_{r} \cos \left(x / l_{0}\right)$,

where the amplitude $A=0.001$ is chosen to be small to approximate the linear regime. The parameter $v$ determines the radial scale of the perturbation. In the case of a straight loop an exact solution can be calculated by solving the dispersion relation to obtain the phase speed. In our simulations we do not have such a solution for our geometry and instead choose $v=2$ which provides a perturbation of the appropriate spatial scale to efficiently excite oscillations. We note the velocity perturbation is only defined within the range $-\pi / 2<x / l_{0}<\pi / 2$ consistent with the loop location (see Fig. 9).

\subsection{Period ratios}

Numerical simulations were performed for velocity perturbations with $n=1,2$, and 3 . In each case the density perturbation was recorded at several points inside the coronal loop and analysed using a periodogram to obtain the period/s of oscillation present. The results of the numerical simulations are summarised in Fig. 10 and Table 1.

The conversion of dimensionless numerical results to physical units is done by choosing appropriate normalisation 
constants for speed $\left(v_{0}\right)$, time $\left(t_{0}\right)$, and length $\left(l_{0}=v_{0} t_{0}\right)$. Our normalisation is based on the observations of Nakariakov et al. (2003). The loop is estimated as $L=25 \mathrm{Mm}$ (taken to be the length along its axis) which defines our normalising length scale $\left(l_{0}=8.79 \mathrm{Mm}\right)$. For the observation, the external Alfvén speed was estimated in the range $4.5-5.3 \mathrm{Mm} / \mathrm{s}$. For convenience, we choose a normalising speed of $4.36 \mathrm{Mm} / \mathrm{s}$ which is slightly lower but gives a period of oscillation for the global mode $P_{1}$ that is equal to the analytical solution for a straight slab (with the same density contrast and aspect ratio at the apex). We note that the choice of normalising constant has no effect on the calculated period ratios. It is then apparent from Table 1 that our curved and expanding loop geometry has the effect of modifying the periods of oscillation such that the period ratios are greater than the case of a straight loop. For each period ratio the modification is $\gtrsim 5 \%$.

Figure 10 shows the spectral power of oscillations excited by drivers with different values of $n$ (see Eq. (10)). The lines have been normalised for visibility. The driver with $n=1$ (solid line) excites the global mode alone. The $n=3$ driver (red dashed line) excites the global and $n=5$ modes in addition to $n=3$. The driver for $n=2$ does not excite any odd modes but also weakly excites $n=4$. We note that the frequency of the global mode is the same whether excited by a driver with $n=1$ or 3 , as expected. The vertical dotted lines correspond to the frequencies for harmonics $n=1-5$ calculated for a straight loop, which are equal for $n=1$ by our choice of normalisation constants.

\subsection{Spatial structure}

Next we consider the spatial profiles of the standing modes. For our curved loop we have a variation in parameters in the longitudinal direction, and so can expect the modes to be modified accordingly. In Figs. 11 and 12, the crosses show the oscillation amplitude along the loop axis for the different longitudinal harmonics in Table 1. For each harmonic, a periodogram routine was used to calculate the spectral power at the particular (fixed) frequency. When calculating the spectral amplitude as the square root of the power, the sign is added by hand for comparison with the dashed sinusoidal profiles corresponding to the solutions for a straight loop. The effect of the curved and expanding loop geometry causes a shift of anti-nodes towards the loop apex. For $n>2$ there is also a decrease in the amplitude of anti-nodes near the loop apex compared with those near the loop footpoints.

\subsection{Dependence on stratification height}

The modification of the spatial profiles and increase in period ratio is qualitatively similar to that seen by Pascoe et al. (2009b) in their model of a straight loop with an expanding cross-section (in particular the $n=1$ and $n=3$ modes also considered by those authors). In the model presented in this paper the potential magnetic field links the curvature and expansion of the loop. The expansion at the apex being common to both these studies suggests this feature may be more important than the curvature. The model of Pascoe et al. (2009b) also includes the longitudinal variation of the internal Alfvén speed, in contrast to the constant internal Alfvén speed in the current model. To investigate this effect, Fig. 13 shows the effect of varying the vertical stratification paramater $\Lambda$ (see Eq. (9)). Increasing the value of $\Lambda$ above 0.5 causes the Alfvén speed to vary along the loop axis, becoming smaller at the loop apex than at the loop footpoints, as shown in the top panel of Fig. 13. The effect of a lower average
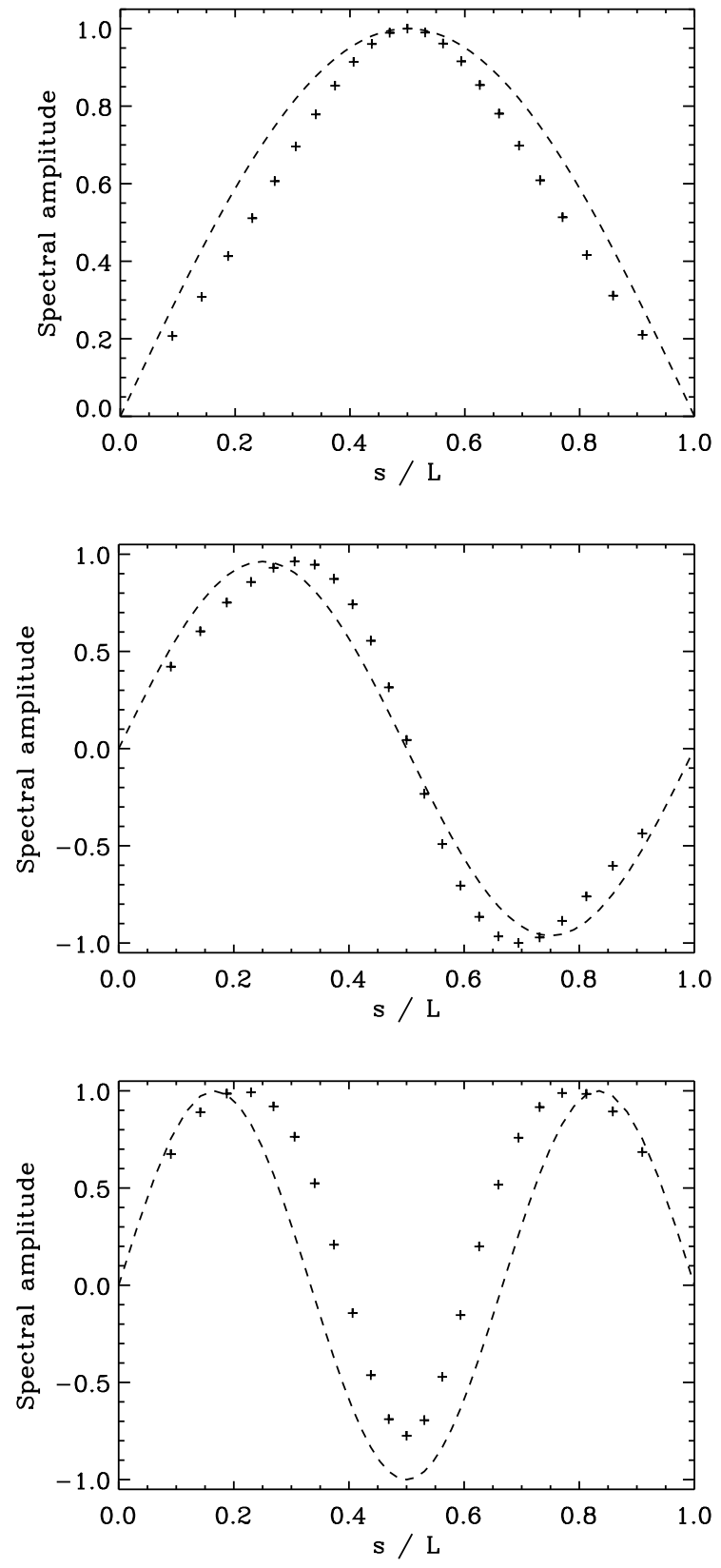

Fig. 11. Oscillation amplitude along the axis of a curved coronal loop for the $n=1$ (top), 2 (middle), and 3 (bottom) sausage modes. The dashed lines show the sinusoidal profiles corresponding to the solutions for a straight loop.

Alfvén speed causes the periods of the harmonics to increase (middle panel) although the period ratios remain roughly constant (bottom panel).

\section{Discussion and conclusions}

The spatial profile of the standing modes is modified by the curved and expanding loop geometry. In particular we see that the $n=3$ longitudinal harmonic has an anti-node at the loop apex which is smaller in amplitude and spatial extent than the sinusoidal profile for a straight loop. On the other hand the spatial extent of the anti-nodes at the loop legs is larger than for a straight loop. This suggests the possibility that observations with a low spatial resolution could more easily confuse the $n=2$ and $n=3$ standing modes in curved (and expanding) coronal loops. 

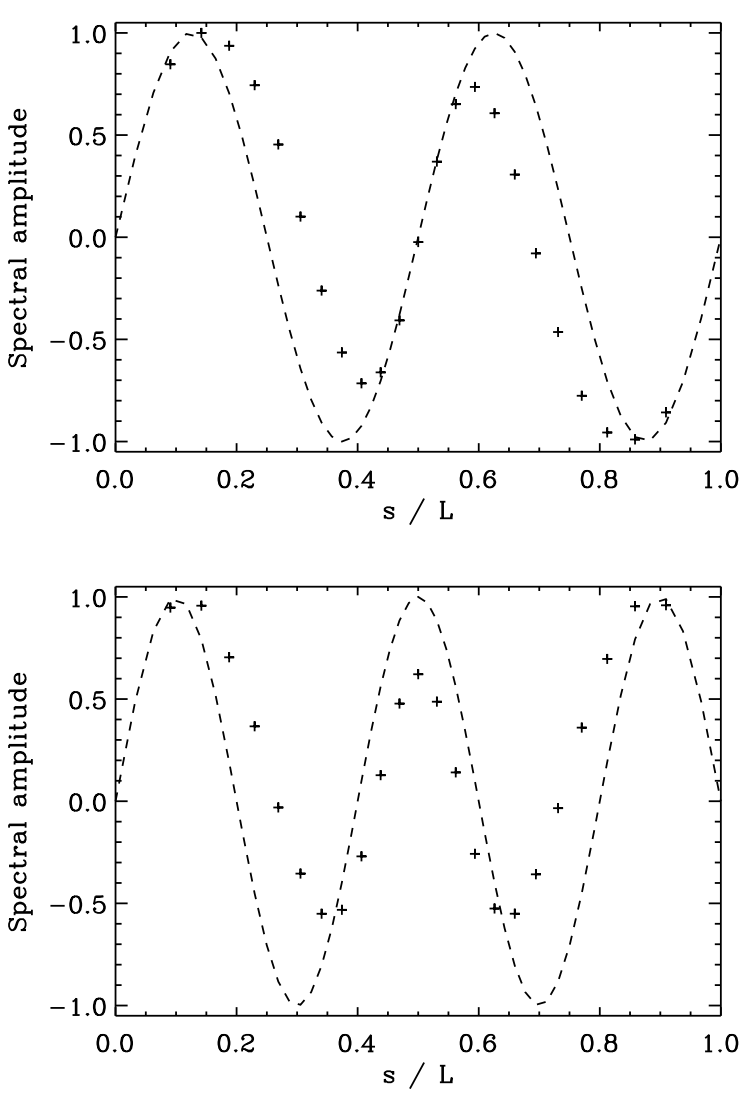

Fig. 12. Same as Fig. 11 but for modes $n=4$ (top) and $n=5$ (bottom).

In particular, we reconsider the observations of Nakariakov et al. (2003) who report the observation of sausage modes at three spatial locations. Their interpretation is in terms of the $n=1$ and $n=2$ harmonics. The global $(n=1)$ mode is identified as having a larger spectral amplitude near the loop apex than near the loop footpoints. This spatial distribution is reproduced by any three observations points along the loop so long as the central observation point is indeed the one closest to the loop apex. The $n=2$ mode is identified by having a lower spectral amplitude near the loop apex than near the loop footpoints. We have demonstrated with our results that this spatial dependence may also be satisfied by an $n=3$ mode in a curved an expanding coronal loop.

To further illustrate this point Fig. 14 shows the amplitudes of the $n=1$ and $n=3$ modes from our numerical simulations (see Fig. 11) as solid lines (here we ignore the sign for $n=3$ ). The vertical dashed lines correspond to observation points which would reproduce qualitatively the spatial dependence of the two periodicities reported by Nakariakov et al. (2003). For the observations at "Foot 1", "Loop top" and "Foot 2" the longer period mode has a ratio of spectral amplitudes of approximately 0.8:1:0.6, while the shorter period mode has a ratio of 1:0.5:0.8. Taking the vertical dashed lines to be the location of these three observation points gives ratios of spectral amplitudes of 0.6:1:0.3 for $n=1$ and 1:0.5:0.9 for $n=3$. As proposed by Melnikov et al. (2005), we therefore consider the possibility of the shorter period mode being the $n=3$ standing mode rather than $n=2$, having demonstrated with our numerical simulations that we can qualitatively reproduce the spatial dependence of the observed oscillations. A more precise account of the spatial dependence would require a full 3D simulation with forwardmodelled radio emission.
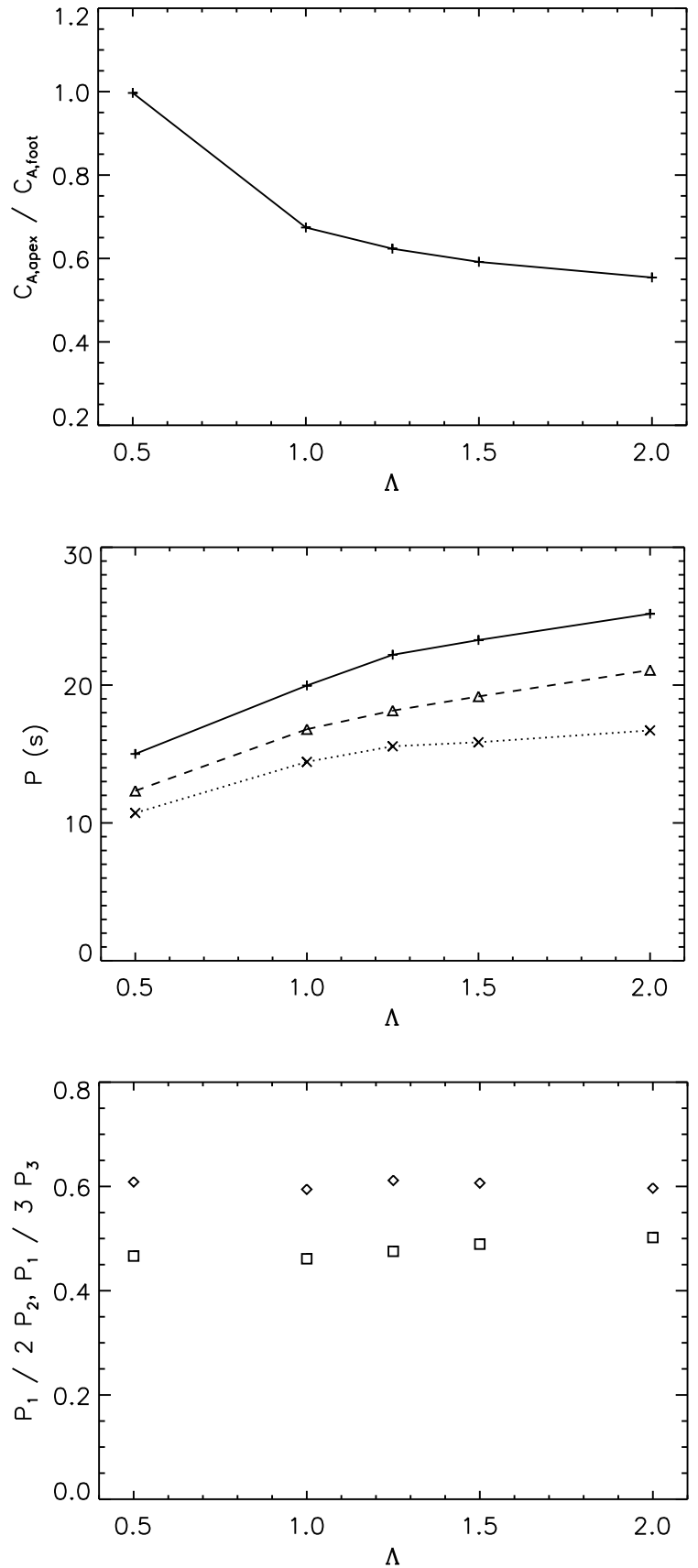

Fig. 13. Dependence of loop parameters as a function of the density stratification parameter $\Lambda$. Top: ratio of Alfvén speed at the loop apex and loop footpoint. Middle: period of oscillation; $P_{1}, P_{2}$, and $P_{3}$ are represented by plus signs, triangles and crosses, respectively. Bottom: period ratios $P_{1} / 2 P_{2}$ (triangles) and $P_{1} / 3 P_{3}$ (squares).

Nakariakov et al. (2003) report $P_{1}$ of 14-17 s and $P_{2}$ of $8-11 \mathrm{~s}$ and so the period ratio $P_{1} / 2 P_{2}$ is approximately $0.64-1.06$ with an average of 0.8 . In comparison the numerical simulations and straight loop estimates give 0.61 and 0.58 , respectively, for $P_{1} / 2 P_{2}$ (Table 1 ). If instead we consider the shorter period mode to be $n=3$ then we can calculate the observational period ratio $P_{1} / 3 P_{3}$ of approximately $0.42-0.71$ with an average of 0.53 . This compares more favourably with 0.47 for our numerical simulations and 0.44 for the straight loop analytical estimate. The interpretation using $n=3$ therefore more readily accounts for the large ratio of the two observed periodicities. For comparison, to account for such a period ratio without 


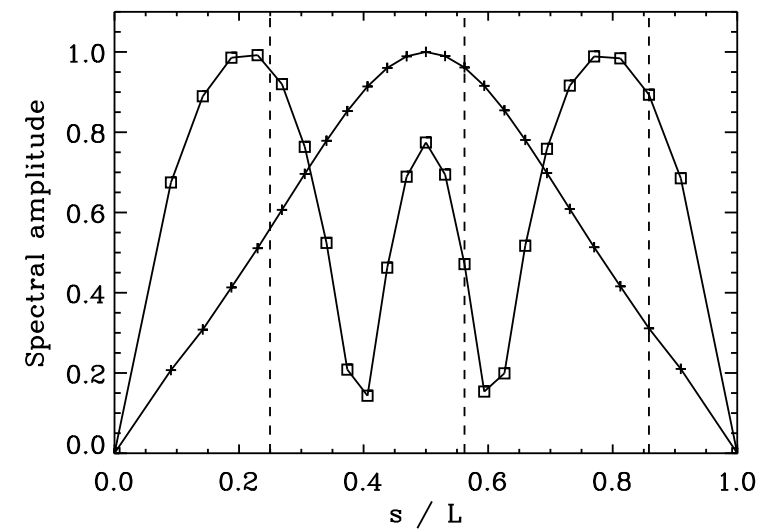

Fig. 14. Spatial dependence (solid lines) of the $n=1$ and $n=3$ standing sausage modes in a curved coronal loop (see Fig. 11). The vertical dashed lines represent three points of constant separation which qualitatively reproduce the spatial dependence of the oscillations observed by Nakariakov et al. (2003).

introducing curvature we can consider the dependence on the loop aspect ratio calculated by Macnamara \& Roberts (2011) and described in Sect. 2.1. Figure 3 shows that $P_{1} / 3 P_{3} \approx 0.53$ requires $a / L \approx 0.2$, which is approximately twice that which is observed by Nakariakov et al. (2003).

We have shown that the effect of our curved and expanding loop acts to increase the period ratios in comparison with those for a straight loop. In the case of a straight loop a 3D (cylindrical) rather than 2D (slab) geometry causes a decrease in the period ratios (e.g. Fig. 2). We might expect a similar decrease for the period ratios of 3D curved coronal loops compared with our 2D simulations. On the other hand, the effect of expansion on the standing mode would also be greater in 3D than in $2 \mathrm{D}$ and this effect might prove to be stronger, causing the period ratios to increase further rather than decrease. Additionally, for straight loops the sausage mode is weakly dependent on the choice of slab or cylindrical geometry (e.g. Edwin \& Roberts 1982, 1983). However, this does not necessarily remain once curvature is introduced, which has the effect of breaking the azimuthal symmetry.

An additional factor that might favour the interpretation using $n=3$ concerns the symmetry of the initial perturbation and the selectivity of the different standing modes. The $n=1$ and $n=3$ are both excited in the case of a perturbation with odd symmetry such as one localised near the loop apex (e.g. Fig. 10). This perturbation would only weakly excite the $n=2$ and other even harmonics. Since we assume in any case the $n=1$ mode is excited, it is reasonable that the $n=3$ mode should also be excited to some extent. This is particularly true in the case of expanding loops increases the coupling between harmonics (Pascoe et al. 2009b). For the $n=1$ and $n=2$ modes to be exited simultaneously requires a driver that excites both odd and even modes (with standing modes $n>2$ apparently being too weak to be observed) such as one which is significantly offset from the loop apex (e.g. Pascoe et al. 2009a, for the case of standing kink modes).

In addition to the event discussed in detail in this paper, another multi-periodic observation was reported by Inglis \& Nakariakov (2009). NoRH observations of a solar flare on 3 July 2002 revealed oscillations with periods of approximately $28 \pm 2,18 \pm 1$, and $12 \pm 1 \mathrm{~s}$. The observations were not resolved spatially, although the two longest periods were also observed in RHESSI data. Taking these periods to be $P_{1}$,
$P_{2}$, and $P_{3}$, respectively, gives the ratios $P_{1} / 2 P_{2} \approx 0.78$ and $P_{1} / 3 P_{3} \approx 0.78$. These ratios are larger than would be expected even taking into account the effects discussed in this paper. Inglis \& Nakariakov (2009) proposed an interpretation of the observations in terms of magnetic reconnection being periodically triggered by kink mode oscillations. We may again reconsider these periods in terms of higher order (odd) harmonics. If the periods correspond to $P_{1}, P_{3}$, and $P_{5}$ the ratios (Table 1) would be expected to be $P_{1} / 3 P_{3} \approx 0.47$ and $P_{1} / 5 P_{5} \approx 0.35$. If we take into account the errors reported by Inglis \& Nakariakov (2009) on the observed periods, the equivalent observational ratios could be as low as 0.46 and 0.40 , respectively. The ratio for the shortest period oscillation therefore still remains significantly higher than expected for $P_{1} / 5 P_{5}$.

Acknowledgements. This work is supported by the Marie Curie PIRSES-GA2011-295272 RadioSun project, the European Research Council under the SeismoSun Research Project No. 321141 (DJP, VMN). The computational work for this paper was carried out on the joint STFC and SFC (SRIF) funded cluster at the University of St Andrews (Scotland, UK).

\section{References}

Andries, J., Arregui, I., \& Goossens, M. 2005, ApJ, 624, L57

Andries, J., van Doorsselaere, T., Roberts, B., et al. 2009, Space Sci. Rev., 149, 3

Arber, T., Longbottom, A., Gerrard, C., \& Milne, A. 2001, J. Comput. Phys., 171,151

Arregui, I., Oliver, R., \& Ballester, J. L. 2004, A\&A, 425, 729

Aschwanden, M. J., Fletcher, L., Schrijver, C. J., \& Alexander, D. 1999, ApJ, 520,880

Aschwanden, M. J., Nakariakov, V. M., \& Melnikov, V. F. 2004, ApJ, 600, 458

Brady, C. S., Verwichte, E., \& Arber, T. D. 2006, A\&A, 449, 389

Cadez, V. M., Oliver, R., \& Ballester, J. L. 1996, A\&A, 314, 636

Cally, P. S. 1986, Sol. Phys., 103, 277

Cooper, F. C., Nakariakov, V. M., \& Williams, D. R. 2003, A\&A, 409, 325

Díaz, A. J., Donnelly, G. R., \& Roberts, B. 2007, A\&A, 476, 359

Donnelly, G. R., Díaz, A. J., \& Roberts, B. 2006, A\&A, 457, 707

Donnelly, G. R., Díaz, A. J., \& Roberts, B. 2007, A\&A, 471, 999

Edwin, P. M., \& Roberts, B. 1982, Sol. Phys., 76, 239

Edwin, P. M., \& Roberts, B. 1983, Sol. Phys., 88, 179

Edwin, P. M., Roberts, B., \& Hughes, W. J. 1986, Geophys. Res. Lett., 13, 373

Erdélyi, R., \& Verth, G. 2007, A\&A, 462, 743

Goossens, M., \& Hollweg, J. V. 1993, Sol. Phys., 145, 19

Goossens, M., Terradas, J., Andries, J., Arregui, I., \& Ballester, J. L. 2009, A\&A, 503,213

Gruszecki, M., \& Murawski, K. 2008, A\&A, 487, 717

Gruszecki, M., Murawski, K., \& McLaughlin, J. A. 2008, A\&A, 489, 413

Inglis, A. R., \& Nakariakov, V. M. 2009, A\&A, 493, 259

Inglis, A. R., van Doorsselaere, T., Brady, C. S., \& Nakariakov, V. M. 2009, A\&A, 503, 569

Jelínek, P., \& Karlický, M. 2012, A\&A, 537, A46

Jelínek, P., Karlický, M., \& Murawski, K. 2012, A\&A, 546, A49

Katsiyannis, A. C., Williams, D. R., McAteer, R. T. J., et al. 2003, A\&A, 406, 709

Li, B., Habbal, S. R., \& Chen, Y. 2013, ApJ, 767, 169

Li, B., Chen, S.-X., Xia, L.-D., \& Yu, H. 2014, A\&A, 568, A31

Liu, W., Ofman, L., Nitta, N. V., et al. 2012, ApJ, 753, 52

Lomineishvili, S. N., Zaqarashvili, T. V., Zhelyazkov, I., \& Tevzadze, A. G. 2014, A\&A, 565, A35

Luna-Cardozo, M., Verth, G., \& Erdélyi, R. 2012, ApJ, 748, 110

Macnamara, C. K., \& Roberts, B. 2011, A\&A, 526, A75

McEwan, M. P., Donnelly, G. R., Díaz, A. J., \& Roberts, B. 2006, A\&A, 460 , 893

McEwan, M. P., Díaz, A. J., \& Roberts, B. 2008, A\&A, 481, 819

Melnikov, V. F., Reznikova, V. E., Shibasaki, K., \& Nakariakov, V. M. 2005, A\&A, 439, 727

Murawski, K., \& Roberts, B. 1994, Sol. Phys., 151, 305

Nakariakov, V. M., \& Roberts, B. 1995, Sol. Phys., 159, 399

Nakariakov, V. M., Ofman, L., Deluca, E. E., Roberts, B., \& Davila, J. M. 1999,

Science, 285, 862

Nakariakov, V. M., Melnikov, V. F., \& Reznikova, V. E. 2003, A\&A, 412, L7

Nakariakov, V. M., Hornsey, C., \& Melnikov, V. F. 2012, ApJ, 761, 134 
Nisticò, G., Pascoe, D. J., \& Nakariakov, V. M. 2014, A\&A, 569, A12

Oliver, R., Murawski, K., \& Ballester, J. L. 1998, A\&A, 330, 726

Oliver, R., Ruderman, M. S., \& Terradas, J. 2014, ApJ, 789, 48

Pascoe, D. J. 2014, Res. Astron. Astrophys., 14, 805

Pascoe, D. J., Nakariakov, V. M., \& Arber, T. D. 2007a, Sol. Phys., 246, 165

Pascoe, D. J., Nakariakov, V. M., \& Arber, T. D. 2007b, A\&A, 461, 1149

Pascoe, D. J., de Moortel, I., \& McLaughlin, J. A. 2009a, A\&A, 505, 319

Pascoe, D. J., Nakariakov, V. M., Arber, T. D., \& Murawski, K. 2009b, A\&A, 494, 1119

Pascoe, D. J., Nakariakov, V. M., \& Kupriyanova, E. G. 2014, A\&A, 568, A20

Pascoe, D. J., Goddard, C. R., \& Nakariakov, V. M. 2016, A\&A, 593, A53

Roberts, B., Edwin, P. M., \& Benz, A. O. 1983, Nature, 305, 688

Roberts, B., Edwin, P. M., \& Benz, A. O. 1984, ApJ, 279, 857

Shen, Y., \& Liu, Y. 2012, ApJ, 753, 53

Smith, J. M., Roberts, B., \& Oliver, R. 1997, A\&A, 317, 752

Srivastava, A. K., Zaqarashvili, T. V., Uddin, W., Dwivedi, B. N., \& Kumar, P. 2008, MNRAS, 388, 1899
Terradas, J., Oliver, R., \& Ballester, J. L. 1999, ApJ, 517, 488 Terradas, J., Oliver, R., \& Ballester, J. L. 2006, ApJ, 650, L91

van Doorsselaere, T., Verwichte, E., \& Terradas, J. 2009, Space Sci. Rev., 149, 299

Vasheghani Farahani, S., Hornsey, C., Van Doorsselaere, T., \& Goossens, M. 2014, ApJ, 781, 92

Verth, G., \& Erdélyi, R. 2008, A\&A, 486, 1015

Verth, G., Van Doorsselaere, T., Erdélyi, R., \& Goossens, M. 2007, A\&A, 475, 341

Verth, G., Erdélyi, R., \& Goossens, M. 2010, ApJ, 714, 1637

Verth, G., Goossens, M., \& He, J.-S. 2011, ApJ, 733, L15

Verwichte, E., Nakariakov, V. M., Ofman, L., \& Deluca, E. E. 2004, Sol. Phys., 223, 77

Verwichte, E., Foullon, C., \& Nakariakov, V. M. 2006a, A\&A, 446, 1139

Verwichte, E., Foullon, C., \& Nakariakov, V. M. 2006b, A\&A, 449, 769

Verwichte, E., Foullon, C., \& Nakariakov, V. M. 2006c, A\&A, 452, 615

Yuan, D., Shen, Y., Liu, Y., et al. 2013, A\&A, 554, A144

Zaitsev, V. V., \& Stepanov, A. V. 1982, Sov. Astron. Lett., 8, 132 